\title{
Research on synchronization algorithm for high speed terminal OFDM system in space and earth integrated network
}

\author{
An Chenyi ${ }^{1, a}$ \\ ${ }^{1}$ School of Electrical and Electronic Engineering, Huazhong University of Science and Technology, \\ Wuhan 430074, China \\ aanchenyi1705@126.com
}

\begin{abstract}
Focus on low signal-to-noise ratio and reliability of terminals with high speed, this paper puts forward the study on synchronization algorithm for high-speed terminal OFDM system in space and earth integrated network. We build a simulation platform of OFDM system high-speed channel, to test the performance of bit error with moving speed as $50 \mathrm{~km} / \mathrm{h}, 100 \mathrm{~km} / \mathrm{h}$ and $250 \mathrm{~km} / \mathrm{h}$. The simulations result show that with the increase of speed, the performance of the system have declined, but increasing the signal-to-noise ratio can improve the performance of the system. When the SNR is higher than $2 \mathrm{~dB}$, the error rate has near performance. Therefore, proposed synchronization algorithm can resist the Doppler effect of high speed to a certain extent.
\end{abstract}

Keywords: Component, formatting, style, styling, insert

\section{Introduction}

As an important national information infrastructure, integrated information network is of great significance to the development of national interests, national security, which also is beneficial to the people's livelihood and promote economic development. It can realize the information of global coverage, broadband transmission, and civil military integration. Since the Internet by the only way, it must be passed China's information network.

The air space ground integrated information network is formed by a plurality of different tracks, different types, and different properties of the satellite constellation of global coverage. Through inter and ground satellite link ground, users in sea and deep air, as well as a variety of aircraft communication platform combined with IP as information intensive. Using intelligent high speed satellite processing, switching and routing technology, we can get best utilization between the optical and infrared multi spectral information, information resources. Integration of large capacity high-speed broadband information network can obtain accurate and fast processing and efficient transmission of information, namely, space-based air ground integration network.

In the process of network integration, integration of signal system should be given priority. Orthogonal frequency division multiplexing (OFDM) is a high-speed transmission technology in wireless environment, which is suitable for high-speed data transmission in wireless communication channel with multipath propagation and Doppler shift. It can effectively combat multipath effect, eliminate inter-symbol interference. What's more, it can resist frequency selective fading, and high channel utilization. Therefore, if the OFDM information is used in the integrated information network, it will greatly improve the efficiency of bandwidth utilization.

Synchronization is one of the key technologies in the OFDM system. The transmitter and receiver clock have oscillator instability and the channel of the Doppler effect, so the sender and the receiver carrier frequency show a certain deviation. Those destroy the orthogonality between subcarriers, resulting in inter carrier interference is serious. If the timing estimation not exactly, makes FFT window containing two adjacent symbols.

The domestic and foreign research institutions have done a lot of researches on OFDM synchronization technology. Guo Yi proposed cooperative diversity in OFDM system and frequency synchronization, using time domain conjugate symmetry training sequence to complete the coarse timing synchronization. The received signal and the local time training sequence segmented moving related fine timing synchronization. Wang Yaxin put forward the research of timing synchronization in OFDM system, which improved search algorithm based on high precision 2-D. It applies CP edge in multipath fading channel search synchronization. Through the design of new timing metric, $2 \mathrm{~N}$ algorithm has better complex degree conditions than those of synchronous effect on search algorithm. You Zhihong proposed a timing synchronization algorithm based on compressed sensing. He designed a synchronization metric function based on the sequence symmetry, which can be used to reduce the length of the synchronization sequence by observing the synchronization sequence in the OFDM. Wu Di put forward the synchronization technique of CO-OFDM system based on training sequence, the sequence of constant amplitude 
zero correlation constitutes the synchronous signal, without increasing the complexity of the algorithm. Timing metric function has a sharp peak at the right time, accurate timing estimation.

The existing OFDM synchronization technology has the problem of low estimation accuracy and limited frequency estimation range. It results in an integrated information network, especially in the presence of severe Doppler shift of the high-speed mobile channel. Those reduces the communication SNR and reliability.

Focus on low signal-to-noise ratio and reliability of terminals with high speed, this paper puts forward the study on synchronization algorithm for high-speed terminal OFDM system in space and earth integrated network. We build a simulation platform of OFDM system high-speed channel, to test the performance of bit error with moving speed as $50 \mathrm{~km} / \mathrm{h}, 100 \mathrm{~km} / \mathrm{h}$ and $250 \mathrm{~km} / \mathrm{h}$. The simulations result show that with the increase of speed, the performance of the system have declined, but increasing the signal-to-noise ratio can improve the performance of the system. When the SNR is higher than $2 \mathrm{~dB}$, the error rate has near performance. Therefore, proposed synchronization algorithm can resist the Doppler effect of high speed to a certain extent.

\section{Basic principle and development of OFDM}

As a multi-carrier modulation technique for multiplexing, the basic principle of OFDM is that the high-speed communication flow through the string and conversion into a multiplex low speed data stream. The modulated transmission is respectively carried out in orthogonal carrier spacing equal to improve the ability of anti-multipath interference and anti-fading. By setting the sub carrier spacing, the frequency spectrum of the subcarriers is overlapped and orthogonal. To improve the spectrum utilization and eliminate the influence of inter-symbol interference. The orthogonal modulation and demodulation in each sub channel can be realized by IFFT and FFT, which reduces the complexity of OFDM implementation. OFDM system schematic diagram shown in figure 1.

After encoding the input system, baseband information bit stream is through the multi-band signal mapping, string and transform form $\mathrm{N}$ branches of each branch signal by inverse Fourier transform of IFFT. It is modulated to the $\mathrm{N}$ sub channel, and then through the string transform, inserting cyclic prefix, analog-to-digital conversion, carrier the modulation process low pass filter by sending RF amplifier. The receiving end receives the data and then carries out the corresponding inverse transform to demodulate the baseband information bit. At the same time, it is often used in the receiver to improve the performance of OFDM system, which is used to eliminate the timing offset and carrier frequency offset.

\section{Synchronization problem in OFDM system}

If there is frequency deviation, single carrier system will make the received signal to produce a certain attenuation and phase rotation. Unfavorable influence of course will cut through the equalizer to eliminate. For multi-carrier system, the influence of carrier frequency offset is much more serious than that of single carrier system. Especially in the OFDM system, it is required that each subcarrier is orthogonal to each other. If there is a frequency shift, the orthogonality is severely damaged.

Therefore, OFDM system for carrier synchronization requirements are relatively high. There are three requirements in OFDM system.

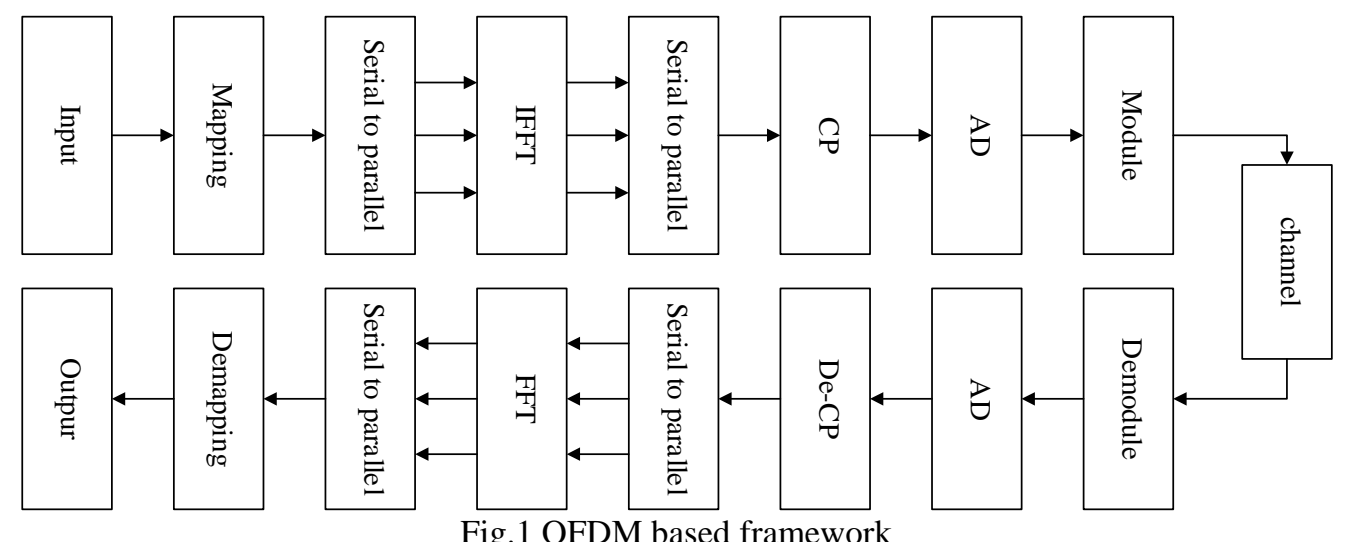

1) Carrier synchronization: the oscillation frequency of the receiver is the same as the frequency of the transmitted carrier.

2) Symbol synchronization: IFFT and FFT start and stop times are the same.

3) Sample synchronization: receiver and transmitter sampling frequency.

In the OFDM system, the receiver must accurately determine the starting position of an OFDM symbol, based on the removal of protection interval and obtain complete symbol data blocks the correct completion of FFT operations. This is the OFDM symbol timing synchronization. For OFDM system with cyclic prefix, symbol-timing interval can be positioned to 
protect; and not for the cyclic prefix symbol structure. In the multipath environment, in order to prevent inter carrier interference and obtain the best performance of the system, still need to strictly determine the best timing position, the symbol timing offset on the system impact analysis as follows Figure 2 is a schematic diagram, symbol timing deviation.

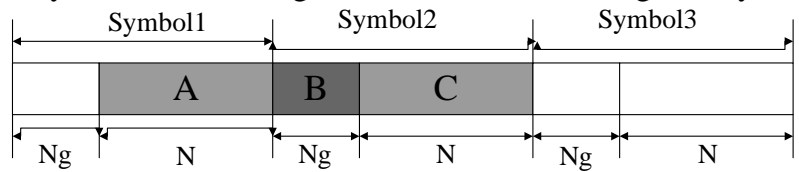

Fig.2 Symbol timing deviation

\section{OFDM synchronization algorithm in high speed motion environment}

\subsection{Timing synchronization improvement}

The last chapter analysis of several classical synchronization algorithms are based on training sequence. It can be found that synchronous joint has good performance and has a larger range of frequency offset estimation. This system adopts joint synchronization based on training sequence (SC) symbol timing algorithm. Main idea is to delay autocorrelation using before the two training sequence, used for timing synchronization. The system will be delayed algorithm locally relevant cross-correlation algorithm combined. Firstly, according to the determined peak position, a frame arrival finds the correlation peak value in the platform. If each peak interval is equal, then check the last peak value to determine the beginning of the next symbol.

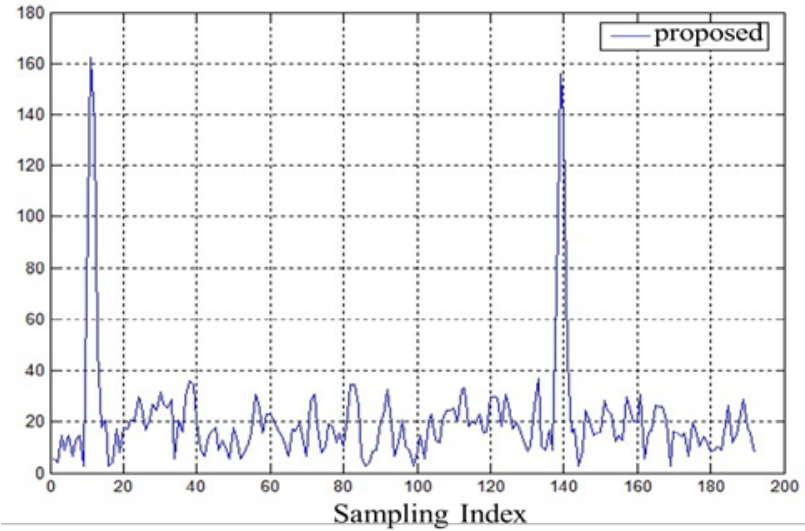

Fig.3 Improved symbol timing correlation output

\subsection{Frequency synchronization Improvement}

For frequency synchronization, this system uses the correlation algorithm. This method requires a training sequence for at least two consecutive repetitions of a leader in the system. Part of the sequences can meet the short training sequence of the system as the frequency offset estimation range of $2000 \mathrm{~Hz}$. The long training sequence in frequency offset estimation range is $250 \mathrm{~Hz}$, a large range of correcting a previous estimate to larger variance. After a small range correction method, the estimation of variance is smaller. Therefore, short training sequence is captured in the range as large as possible. Long training sequence will estimate the range to minimize use of repetitive frequency tracking loop before the suffix to complete. Range of frequency-offset estimation is $125 \mathrm{~Hz}$.

We set 7 level of signal to noise ratio $(-10 \mathrm{~dB},-8 \mathrm{~dB},-4 \mathrm{~dB},-6 \mathrm{~dB},-2 \mathrm{~dB}, 0 \mathrm{~dB}, 2 \mathrm{~dB})$ value of 200 symbols simulation. the system simulation BER curve shown in figure 4.8. It can be seen that the maximum delay of 3 points, the observation time is $0.5 \mathrm{~ms}$. With the increase of speed, the performance of the OFDM system will have a certain degree of decline. But the difference is small. With the increase of the SNR, the performance of the system was improved gradually. When the moving speed is increased from $50 \mathrm{~km} / \mathrm{h}$ to $100 \mathrm{~km} / \mathrm{h}$, the bit error rate of approximately doubling. When increased to $250 \mathrm{~km} / \mathrm{h}$, the error rate increases by about two times. When the SNR is $2 \mathrm{~dB}$, the three bit error rate has speed difference is small 


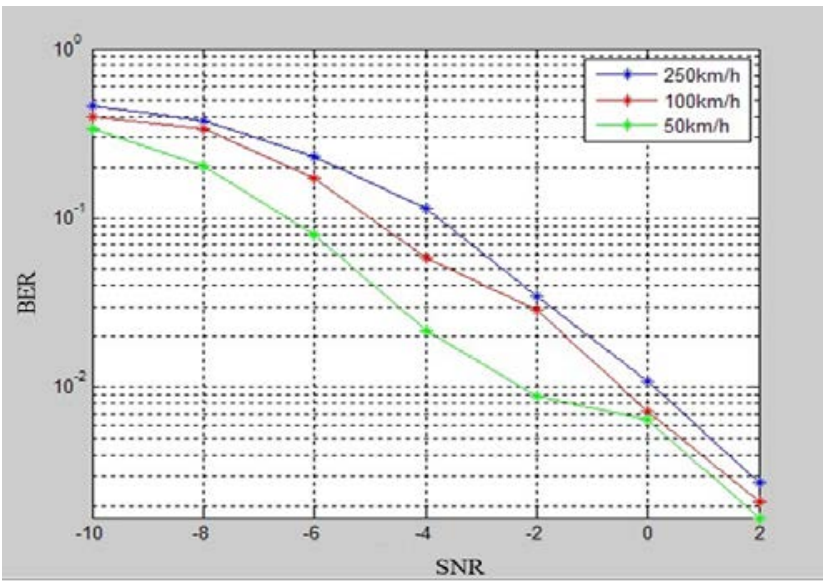

Fig.4 BER of the system under different moving speed

\section{Conclusion}

Focus on low signal-to-noise ratio and reliability of terminals with high speed, this paper puts forward the study on synchronization algorithm for high-speed terminal OFDM system in space and earth integrated network. We build a simulation platform of OFDM system high-speed channel, to test the performance of bit error with moving speed as $50 \mathrm{~km} / \mathrm{h}, 100 \mathrm{~km} / \mathrm{h}$ and $250 \mathrm{~km} / \mathrm{h}$. The simulations result show that with the increase of speed, the performance of the system have declined, but increasing the signal-to-noise ratio can improve the performance of the system. When the SNR is higher than $2 \mathrm{~dB}$, the error rate has near performance. Therefore, proposed synchronization algorithm can resist the Doppler effect of high speed to a certain extent.

\section{References}

[1] Harpreet Kaur,Manoj Kumar,Ajay K. Sharma,Harjit P. Singh. Performance analysis of DWT based OFDM over fading environments for mobile WiMax. Optik - International Journal for Light and Electron Optics, 2016,127(2): 544-547.

[2] Hakan Dogan,Todor Cooklev,James Darabi. Improved low-complexity zero-padded OFDM receivers. Digital Signal Processing, 2016, $51(\mathrm{C}): 92-100$

[3] Gui-Teng Xie,Hong-Yi Yu,Yi-Jun Zhu,Xin-Sheng Ji. A Linear Receiver for Visible Light Communication Systems with Phase Modulated OFDM. Optics Communications, 2016,371:112-116.

[4] Chen Chen,Wen-De Zhong,Dehao Wu. Color Multiplexing Based Unipolar OFDM for Indoor RGB LED Visible Light Communication. Procedia Engineering,2016, $140: 159-165$.

[5] Yue-hai Zhou,F. Tong,Gang-qiang Zhang. Distributed compressed sensing estimation of underwater acoustic OFDM channel. Applied Acoustics,2016, 117:160-166.

[6] Leonid Uryvsky,Serhii Osypchuk. OFDM Signal Research with Varied Subcarriers Number. Transport and Telecommunication Journal,2016, 17(3) :192-201. 